\title{
Navigation System for Omni-directional Automatic Guided Vehicle with Mecanum Wheel
}

\author{
Sulabh Kumra ${ }^{1}$, Rajat Saxena ${ }^{2}$, Shilpa Mehta ${ }^{3}$ \\ ${ }^{1} 22$ Department of Electronics and Instrumentation Engineering, ITM University \\ ${ }^{3}$ Department of Electronics and Communication Engineering, ITM University
}

\begin{abstract}
This paper presents navigation system for an omni-directional AGV (automatic guided vehicle) with Mecanum wheels. The Mecanum wheel, one design for the wheel which can move in any direction, is a conventional wheel with a series of rollers attached to its circumference. The localization techniques for the general mobile robot use basically encoder. Otherwise, they use gyro and electronic compass with encoder. However, it is difficult to use the encoder because in the Mecanum wheel the slip occurs frequently by the rollers attached to conventional wheel's circumference. Hence, we propose the localization of the omnidirectional $A G V$ with the Mecanum wheel. The proposed localization uses encoder, gyro, and accelerometer. In this paper, we ourselves designed and made the AGV with the Mecanum wheels for experiment. And we analyzed the accuracy of the localization when the AGV moves sideways a $20 \mathrm{~m}$ distance at about $20 \mathrm{~cm} / \mathrm{s}$ and $38 \mathrm{~cm} / \mathrm{s}$, respectively. In experimental result, we verified that the accuracies of the proposed localization are $27.4944 \mathrm{~mm}$ and $29.2521 \mathrm{~mm}$ respectively.
\end{abstract}

Keywords: AGV, Mecanum wheel, Omni-directional

\section{INTRODUCTION}

Recently, the interest in the AGV has been increased as the amount of logistics has been increasing. However, the existing AGVs don't work effectively in the narrow workspace because its direction can only change while moving forward or backward. Hence, the interest in the omni-directional AGV is growing, and there are many studies [1-7]. The Mecanum wheels are conventional wheels with a series of rollers attached to their circumference. These rollers have an axis of rotation at $45^{\circ}$ to the plane of the wheel in a plane parallel to the axis of rotation of the wheel. As well as moving forward and backward like conventional wheels, they allow sideways movement by spinning wheels on the front and rear axles in opposite directions. However, it is difficult to use the encoder because in the Mecanum wheel the slip occurs frequently by the rollers attached to conventional wheel's circumference. Hence, we propose the localization of the omni-directional AGV with the Mecanum wheel. The proposed localization uses encoder, gyro and accelerometer.

\section{MEASUREMENT SYSTEM} localization.

In this paper, we designed and made the AGV with the Mecanum wheels for experiment of

The AGV has four Mecanum wheels, four 300W BLDC motors (TM-90D0321), motor drivers which control each of the motors. In addition, we use a laptop for rapid development, and a DAQ (data acquisition) to control the motor driver efficiently. Figure 1 shows the designed omni-directional AGV with Mecanum wheels.

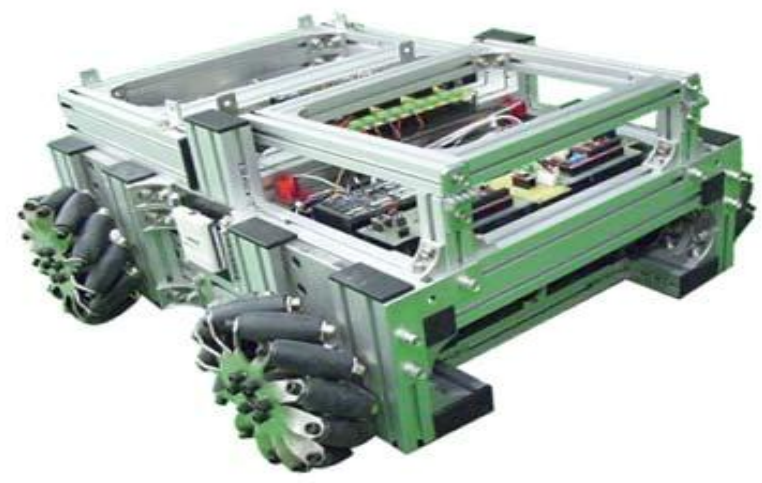

Fig 1. Omni-directional AGV 


\subsection{KINEMATICS}

Figure 2 shows the kinematics of AGV with Mecanum wheel.

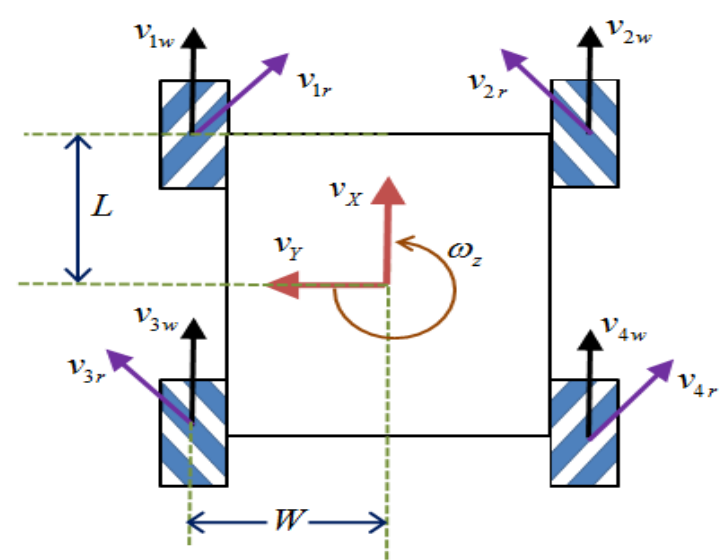

Fig 2. Kinematics of robot with Mecanum wheel

In Figure 2, $\mathrm{L}$ is the distance between the instantaneous center of rotation (ICR) and the center of front wheels or rear wheels. And $\mathrm{W}$ is the distance between front wheels or rear wheels. viw is the linear velocity by rotation of the wheels, and vir is actual force acting on the ground by rollers of Mecanum wheel. Because the rollers have an axis of rotation at $45^{\circ}$ to the plane of the wheel, viX and viY are calculated by viw and vir as follows.

$$
\begin{aligned}
& v_{1 X^{-}}=v_{1 w}+\frac{v_{1 r}}{\sqrt{2}}, v_{1 Y}=\frac{v_{1 r}}{\sqrt{2}} \\
& v_{2 X}=v_{2 w}+\frac{v_{2 r}}{\sqrt{2}}, v_{2 Y}=\frac{v_{2 r}}{\sqrt{2}} \\
& v_{3, X^{\prime}}=v_{3 w}+\frac{v_{3 r}}{\sqrt{2}}, v_{3 Y}=\frac{v_{3 r}}{\sqrt{2}} \\
& v_{4 X^{-}}=v_{4 w}+\frac{v_{4 r}}{\sqrt{2}}, v_{4 Y^{\prime}}=\frac{v_{4 r}}{\sqrt{2}}
\end{aligned}
$$

To summarize the above equation into $\mathrm{v} 1 \mathrm{w}, \mathrm{v} 2 \mathrm{w}, \mathrm{v} 3 \mathrm{w}, \mathrm{v} 4 \mathrm{w}$ is represent by

$$
\left[\begin{array}{c}
v_{1 w} \\
v_{2 w} \\
v_{3 w} \\
v_{4 w}
\end{array}\right]=\left[\begin{array}{ccc}
1 & -1 & -(L+W) \\
1 & 1 & (L+W) \\
1 & 1 & -(L+W) \\
1 & -1 & (L+W)
\end{array}\right]\left[\begin{array}{l}
v_{X} \\
v_{Y} \\
v_{Z}
\end{array}\right]
$$

Here, the above equation represented by a matrix is as follows.

$$
\begin{aligned}
& v_{1 w}=v_{X}-v_{Y}-(L+W) w_{z} \\
& v_{2 w}=v_{X}+v_{Y}+(L+W) w_{z} \\
& v_{3 w}=v_{X}+v_{Y}-(L+W) w_{z} \\
& v_{4 w}=v_{X}-v_{Y}+(L+W) w_{z}
\end{aligned}
$$

That vX, vY and wZ are calculated through the inverse equation as follows.

$$
\begin{gathered}
T=\frac{1}{(L+W)} \\
{\left[\begin{array}{c}
v_{X} \\
v_{Y} \\
w_{Z}
\end{array}\right]=\frac{1}{4}\left[\begin{array}{cccc}
1 & 1 & 1 & 1 \\
-1 & 1 & 1 & -1 \\
-T & T & -T & T
\end{array}\right]\left[\begin{array}{l}
R_{w} \cdot \dot{\theta}_{1} \\
R_{w} \cdot \dot{\theta}_{2} \\
R_{w} \cdot \dot{\theta}_{3} \\
R_{w} \cdot \dot{\theta}_{4}
\end{array}\right]}
\end{gathered}
$$

Where RW and $\Theta i$ are the radius of the wheel and are the rotational speed of each of the wheels, 
Navigation System for Omni-directional Automatic Guided Vehicle with MecanumWheel

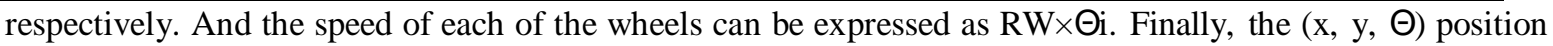
of the AGV can be calculated by accumulated eq. (4).

TABLE 1. Major specifications of sensors

\begin{tabular}{|l|l|l|}
\hline Item (model) & Specification \\
\hline \multirow{2}{*}{$\begin{array}{l}\text { Accelerometer } \\
\text { (myAccel3LV02) }\end{array}$} & Voltage & $3.3 \mathrm{~V}$ \\
\cline { 2 - 3 } & Interface & I2C \\
\cline { 2 - 3 } $\begin{array}{l}\text { Encoder } \\
\text { (TMC-D03) }\end{array}$ & Typ. Range & $2.0 / 6.0 \mathrm{~g}$ \\
\hline \multirow{2}{*}{$\begin{array}{l}\text { Gyro } \\
\text { (myGyro300SPI) }\end{array}$} & Voltage & $5 \mathrm{~V}$ \\
\cline { 2 - 3 } & Resolution & 1000 pulse \\
\cline { 2 - 3 } & Voltage & $5 \mathrm{~V}$ \\
\cline { 2 - 3 } & Interface & SPI \\
\hline
\end{tabular}

\subsection{SENSORS FOR LOCALIZATION}

We use an accelerometer (myAccel3LV02), four encoders (TMC-D03), and a gyro (myGryo300SPI) for the localization of the AGV, and measure them through CAN (controller area network) communication on AT90CAN128 MCU (micro controller unit). Table 1 shows the major specifications of each of the used sensors.

\section{PROPOSED LOCALIZATION}

Figure 3 shows the flowchart of a proposed localization. First, the proposed localization uses information of encoder to resolve the accumulated errors of accelerometer and gyro. Next, each velocity of encoder and gyro are calculated by data from encoder and gyro. And then errors of encoder are calculated through difference between two angular velocities. Next steps are similar to the steps above, but encoder and accelerometer are used.

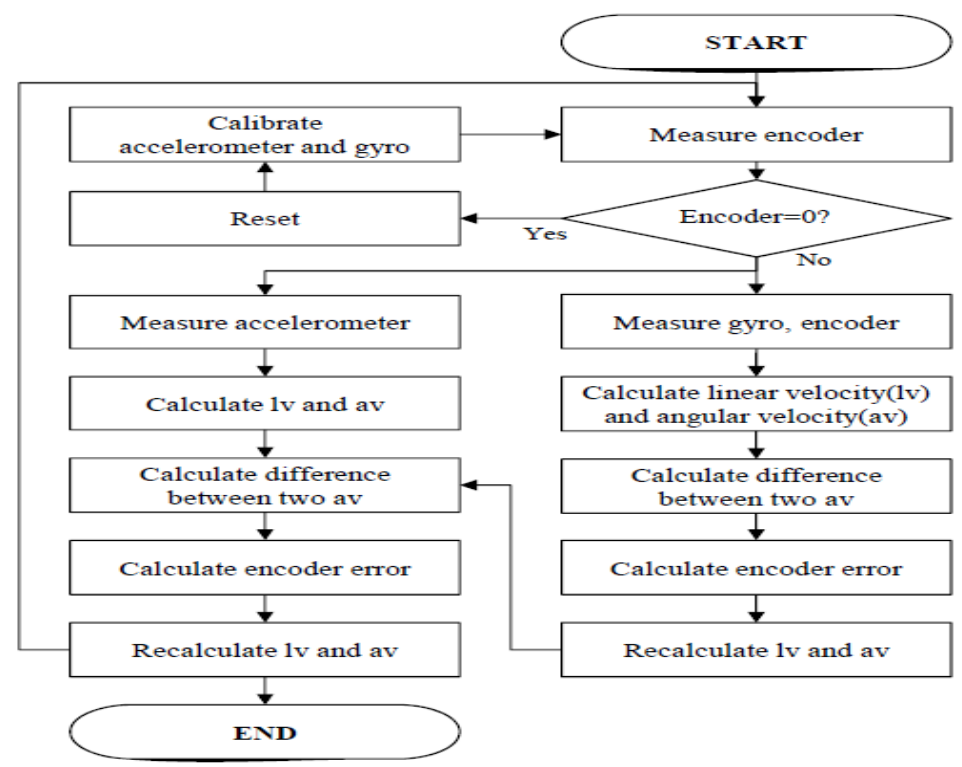

Fig 3. Flowchart of proposed localization

\section{EXPERIMENT}

In the experiment, the performance of the proposed localization is compared with NAV200 when the AGV moves sideways a $20 \mathrm{~m}$ distance at about $20 \mathrm{~cm} / \mathrm{s}$ and $38 \mathrm{~cm} / \mathrm{s}$, respectively. NAV200 which has $\pm 3 \mathrm{~mm}$ accuracy is able to measure very accurate positioning, but is very expensive. Table 2 shows the major specifications of NAV200.

Figure 4 and Figure 5 show representative experimental results, respectively. 


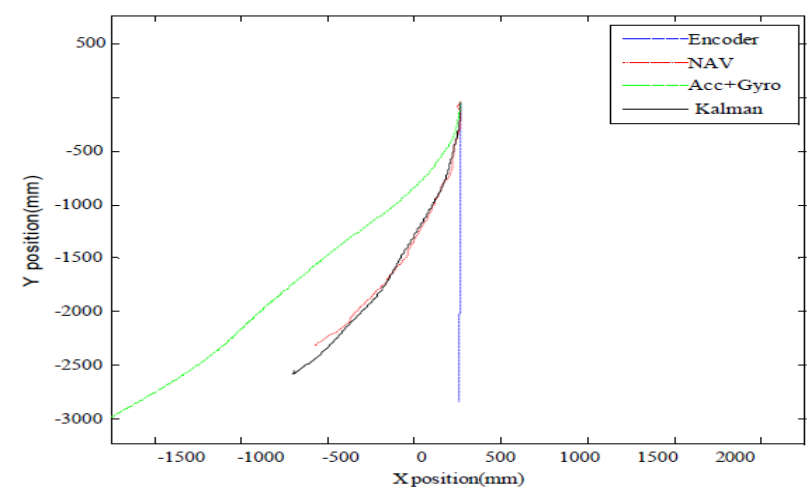

Fig 4. Result of sideways driving $(20 \mathrm{~cm} / \mathrm{s})$

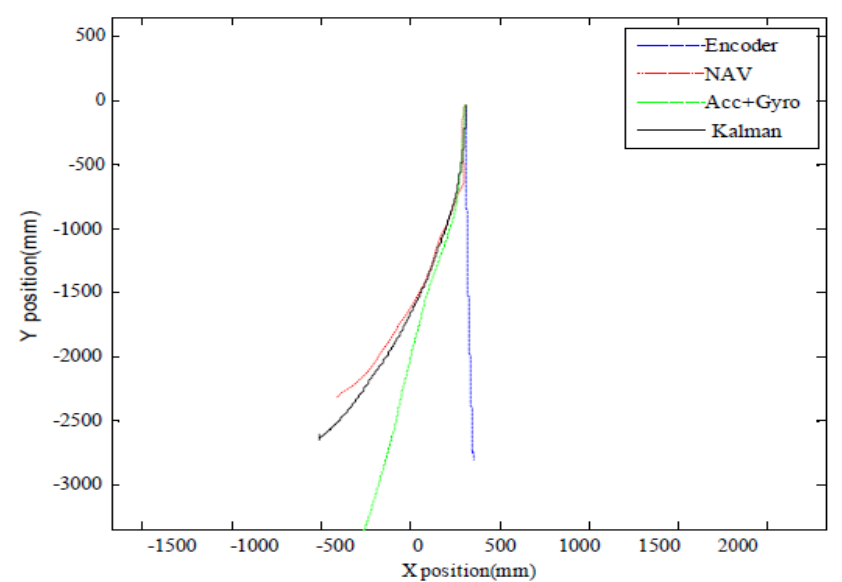

Fig 5. Result of sideways driving $(38 \mathrm{~cm} / \mathrm{s})$

In the result (--) which uses only the encoder, the driving result of the AGV seems like moving straight. But if you see the result (:) using NAV200, we can verify that the AGV don't moved straight by the slip. In case of using accelerometer and gyro, the result (-.) has high errors by the double integral of accelerometer. However, the result (-) of the proposed method is similar to the result of NAV200. Table 2 shows each of the experiments.

TABLE 2. Major specifications of NAV200

\begin{tabular}{|l|l|}
\hline Item & Specification \\
\hline Power supply & $24 \mathrm{~V}$ \\
\hline Operating range & $1.2 \sim 28.5 \mathrm{~m}$ \\
\hline Scanning frequency & $8 \mathrm{~Hz}$ \\
\hline Field of view & $360^{\circ}$ \\
\hline Angular resolution & $0.1^{\circ}$ \\
\hline
\end{tabular}

When the AGV moves sideways at about $20 \mathrm{~cm} / \mathrm{s}$ and $38 \mathrm{~cm} / \mathrm{s}$ (See table 3), the proposed localization is $27.4944 \mathrm{~mm}$ and $29.2521 \mathrm{~mm}$, respectively. And we verify that the proposed localization is effective though sideways driving causes severe slip.

TABLE 3. Experimental results

\begin{tabular}{|l|l|l|}
\hline \multirow{2}{*}{$\#$} & \multicolumn{2}{|l|}{ Specification } \\
\cline { 2 - 3 } & $20 \mathrm{~cm} / \mathrm{s}$ & $38 \mathrm{~cm} / \mathrm{s}$ \\
\hline 1 & 22.7019 & 29.3039 \\
\hline 2 & 34.7432 & 29.4895 \\
\hline 3 & 29.6854 & 43.0997 \\
\hline 4 & 36.6345 & 21.4171 \\
\hline 5 & 34.5811 & 33.8901 \\
\hline Avg. & 27.4944 & 29.2521 \\
\hline
\end{tabular}




\section{CONCLUSION}

This paper presents the localization for an omni-directional AGV with Mecanum wheels. The localization techniques for the general AGV use basically encoder. Otherwise, they use gyro and electronic compass with encoder. However, it is difficult to use the encoder because in the Mecanum wheel the slip occurs frequently by the rollers attached to conventional wheel's circumference. Hence, we propose the localization for the AGV with Mecanum wheels, and it used encoder, gyro, and accelerometer. For experiment, we designed the AGV with the Mecanum wheels, and compared the proposed localization with NAV200 when the AGV moves sideways to $20 \mathrm{~m}$ distance at about $20 \mathrm{~cm} / \mathrm{s}$ and $38 \mathrm{~cm} / \mathrm{s}$, respectively. In experimental results, we verify that the proposed localization is effective though sideways driving causes severe slip.

\section{References}

[1] A. Vis, Survey of research in the design and control of automated guided vehicle systems, Eu- ropean Journal of Operational Research, vol. 170, no. 3, pp. 677-709, 2006.

[2] C. W. Tan and S. S. Park, Design of accelerometer-based inertial navigation systems, IEEE Transactions on Instrumentation and Measurement, vol.54, no. 6, pp. 2520-2030, 2005.

[3] K. L. Han, O. K. Choi, J. W. Kim, H. S. Kim, and J. S. Lee, Design and control of mobile robot with mecanum wheel, ICCASSICE, pp. 2932-2937, 2009.

[4] Dogandzic, J. Riba, G Seco, and A. Lee Swindle-hurst, Positioning and navigation with appli- cations to communications, IEEE Signal Proc. Magazine, vol. 22, no. 4, pp. 10-11, 2005.

[5] W. Tan and S. S. Park, Design of accelerometer-based inertial navigation systems, IEEE Trans-actions on Instrumentation and Measurement, vol.54, pp. 2520-2030, 2005.

[6] J. K. Hwang, M. Uchanski, and C. K. Song, Vehicle speed estimation based on kalman filtering of accelerometer and wheel speed measurements, International Journal of Automotive Tech-nology, vol. 6, no. 5, pp. 475-481, 2005.

[7] Q. Pingping, F. Li, and Z. Xin, Design of inertial navigation system based on micromechanical gyroscope and accelerometer, Control and Decision Conference, pp. 1351-1354, 2009. 